\title{
ORIENTACION PARA CUMPLIR LAS TAREAS ADULTAS
}

\author{
Flory Stella Bonilla
}

La vida no es un problema que hay que resolver. Es algo que hay que vivir lo mejor que se pueda: jEs una obligación y un desafio para el ser humano! Entre las luchas y placeres cotidianos que ello implica, existen asignaciones que debemos cumplir, y que reciben el nombre de tareas de desarrollo porque reflejan grnancias en destrezas motoras, intelectuales, socinles y emocionales (Newman y Newman, 1979). La importancia de tales tareas reside en que marcan la orientación que la persona tiene hecia el mundo en cada momento de su vida, por $\mathbf{l}$ forma como utiliza e integra las destrezas previnmente adquiridas.

Para entender las tareas del desarrollo, usunhmente se elaboran taxonomías que organizan temsticas similares: aquellas que afectan el desorrollo físico, el social, el cognitivo, el morl. Dicho de otro modo, existen tareas de desarrollo cognitivo como el pensamiento concrevo en el nino escolar o la formación de una filoscfín de vida del joven adulto. Existen otras tareas para el desarrollo social, como las que envuetven el cambiante involucramiento del individuo con los demás para desarrollar su propio exito de formar relaciones interpersomales. Tambien hay tareas propias de la esfera fisica, como el desarnollo motor y la coordinacion, en b nit̄ez, o los cambios físicos y el proceso de maduración sexual propio de la puberad. Asi, en cada campo de esta totalidad que es el ser humono, siempre debemos trabajar, cumpliendo asignaciones para poder crecer.

Fraud do que es adulto aquel que es capaz de amar y urbajar. En el vivir adulto estas dos tareas exin inexorablemente unidas, porque uno puede amar su trabajo y trabajar el amor. Ciertamente el tubajo tiene una dimensión afectiva-epresiva y el amor nunca existe sin su componente instrumental. En otras palabras, para estar realmente involucrado con el mundo, el ser humano debe ser enriquecido por ese mundo al mismo tiempo que debe invertir partes de su ser en él. Para lograr esta integración, cada persona necesita cumplir asignaciones específicas según la evolución de su desarrollo personal. ¡Eso es parte de la tarea primordial que es vivir la vida!

\section{1. ¿Qué es una tarea adulta?}

Siguiendo las enseñanzas de la naturaleza, que se organiza en ciclos naturales (verano-invierno, día-noche), se ha dividido la vida humana en estadios para su mejor estudio y comprensión: infancia, niñez, adolescencia, vida adulta y ancianidad.

Cuando el criterio para organizar estas etapas es la intencionalidad de la vida, Frenkel, E.(1968), quien cita a Bühler (1933), encontró cinco periodos que marcan la claridad que vamos alcanzando en esa búsqueda: antes de los 15 no hay claridad, entre los 15 y 25 es tentativa, entre 25 y 45 se definen los objetivos específicamente, entre 45 y 65 se evalúa si esos propósitos se cumplieron, y de los 65 en adelante, se aceptan los resultados con resignación o satisfacción. Podría generalizarse, entonces, que son tareas de la vida adulta, definir objetivos de vida, evaluar si se logran y aceptar su resultado, sea de fracaso o satisfacción.

Se logra la sensación de tener un objetivo vital cuando se cumple un programa diario de trabajo, juego, y todo aquello que dé conciencia de la propia valía, es decir, de tener una misión personal. De ahí la importancia de la Educación para ayudar en la formulación de los fines, en la clarificación del sentido de vida 
y, finalmente, en la búsqueda de estrategias apropiadas para alcanzarlos. Es importante reconocer que todas las tareas de desarrollo proceden básicamente de tres fuentes: maduración corporal, normas culturales y expectativas individuales (Lehr, 1980).

Todo ser humano, entonces, debe cumplir determinadas asignaciones en cada etapa de su desarrollo. Así, el niño juega y al hacerlo, practica, aprende y se separa de los objetos primeros de amor, de modo que empieza a ser él mismo para poder amar después. El adolescente explora también su mundo y el universo con pasión, con fidelidad, para tratar de averiguar quién es, y sentirse más libre, más autónomo, de modo que pueda luego escoger trabajo y actividad, para elegir a quién amar. Es decir, para cumplir con las tareas adultas, es necesario haber adquirido exitosamente hasta las más elementales destrezas de etapas anteriores, como el desarrollo de una relación de apego en la infancia, -el desarrollo del lenguaje en el período preescolar- la experimentación en juegos grupales de la niñez.

Al avanzar en el ciclo de la vida se experimentan nuevas situaciones, lo que demanda reorientación y asumir nuevos roles, nuevas tareas y nuevos derechos. Es decir, parece existir un ritmo en todo el desarrollo humano, que avanza de etapas estables a etapas de progreso y de cambio, cada una con tareas específicas asignadas socialmente, las cuales deben ser cumplidas porque de lo contrario invitamos a que la sociedad nos sancione, aumentando así las tensiones del vivir adulto. Parece, entonces, que lo exclusivo del período adulto reside en que las tareas de esta etapa son más complejas $\mathrm{y}$ hay menos tiempo y oportunidades para repetir intentos, por lo que los errores cuestan más. Sin embargo, también parece que las recompensas son mayores. Es propio que durante los periodos estables de crecimiento, el adulto deberá construir una estructura de vida, y en los periodos de crisis, tendrá que revisar y cuestionar esa estructura que formó previamente (Levinson,1978).

Sabemos que cada persona es única, pero también similar a las demás, de ahí que la condición adulta pueda definirse como un esfuerzo universal en busca de continuidad, aunque también debe haber cambio permanente, pero dentro de un proceso ordenado por el contex- to histórico y sociocultural del lugar donde vive cada persona. El individuo que se limita a seguir una rutina que le parece desagradable, es que no ha alcanzado el objetivo de su vida, y eso le genera vacío. La sensación de tener sentido de vida es algo interno' y da plenitud, porque compromete a la persona para trataŕ de cumplir con sus obligaciones.

Las tareas específicas son cruciales y dan carácter a cada período: cuando una tarea se vuelve predominante en la vida de la persona, empieza una etapa, la cual termina cuando la tarea pierde primacía. En síntesis, un período es definido por las tareas que la persona cumple en él y no por los eventos que en él suceden, como bautizos, matrimonios, nacimientos.

Virtualmente todos los eventos demarcadores son importantes siempre, pero según el momento de la vida en que suceden, responderán al cumplimiento de una tarea o de otra (Sheehy, 1976). Así, un matrimonio puede responder a una tarea de separación de los padres, o a una de formación inicial adulta.

Según diferentes estudiosos del desarrollo humano (Havighurst, 1972; Newman y Newman, 1979; Levinson, 1978) las tareas de vida ocurren en la misma secuencia para todas las personas, pero no son invariables ni presentan cambios estructurales en la personalidad de los individuos. Son más bien puntos de encuentro entre la persona y su medio, pues la interacción de la persona con los demás y la integración que hace en su personalidad, de esa interacción, es determinada por las tareas de desarrollo que realiza.

\section{2. ¿Qué es especifico de la Etapa Adulta?}

Siempre sucede algo nuevo en cada etapa de la vida que deviene de aprendizajes anteriores pero que, al mismo tiempo, es resultado único de la etapa particular de desarrollo. Conforme la persona madura, la organización vital se vuelve cada vez más compleja y abstracta (Piaget). Diferentes teóricos del desarrollo humano comparten esta visión de aumento en la complejidad de las tareas, pero difieren en los temas centrales que asignan a esas tareas (Erikson, 1980; Frenkel, 1968; Kermis, 1986). $\mathrm{El}$ período adulto, que comprende alrededor de cinco décadas (de los 18 a los 65), es la 
época más larga de la vida. Se subdivide en: adulto joven, adulto medio y adulto tardío. Cada subetapa requiere del logro de ciertas tareas que variarán según la cultura y el momento específico que se vive.

Desde el punto de vista de Levinson (1978) la vida adulta se organiza en eras, subdivididas por períodos, cada uno con sus propias tareas de desarrollo. En el "período novicio", son tareas principales del adulto joven, que es el que tiene entre 17 y 40 años:

- Formar un sueño y darle lugar en la estructura de vida.

- Establecer relaciones mentoras.

- Adquirir una ocupación.

- Formar relaciones de amor, matrimonio y familia.

Una vez que este adulto joven ha terminado de explorar y de organizarse en su mundo, también lo cuestiona, y esto se conoce como "la transición de los 30"; luego aparece la "acomodación", en la cual Levinson (1978) destaca dos tareas básicas:

Establecer un nido en la sociedad (construir el nido y buscar los intereses personales de una manera propia).

Trabajar para avanzar (planear, luchar, progresar).

Alrededor de los 40 -edad mágica en que el hombre empieza a juzgar si ha tenido éxito o si fracasó en el logro de sus metas- surge la crisis de transición hacia el adulto medio. Entre las asignaciones de esta época de transformación, Levinson (1978) destaca la tarea de "Hacer elecciones cruciales, para modificar o renunciar al sueño". Al finalizar los 50 aparece una etapa de "culminación" en la que se debe lidiar con la pérdida de energía y a la vez, luchar por mantener la cultura. Este teórico argumenta que debemos renunciar a la autoridad de la familia, reconciliarnos con el propio ser y con el mundo y prepararnos para la muerte.

Kermis (1986) considera que en la mitad de la vida las tareas enfocan las relaciones intergeneracionales y sociales, ya que los jóvenes adultos se preocupan no sólo de su sobrevivencia personal, sino de la sobrevivencia de la especie, mientras que los mayores se interesan más por la calidad de la vida que le dejarán a las nuevas generaciones.

Por otra parte, Peck (1968) describe cuatro tareas básicas para el período de la mitad de la vida: socialización versus sexualización en las relaciones, valoración de la sabiduría sobre la fuerza física, flexibilidad mental versus rigidez y flexibilidad emocional versus empobrecimiento emocional. Para los adultos más viejos, señala la necesidad de tres tareas de ajuste: diferenciación del yo versus preocupación por el rol laboral, trascender la preocupación por el cuerpo versus la preocupación corporal y transcender el yo versus la preocupación por él.

Peck (1968) considera que el asunto central de la vida es lo vocacional, por lo que cree que las personas que se pensionan necesitan redefinir su valía. Opina que esta habilidad para mantener un sentido de indentidad y valor es muy importante dadas las realidades físicas tan inestables de las personas mayores.

Havighurst (1972) por otro lado, señala seis tareas del desarrollo, de modo que las personas que envejecen vayan adaptándose poco a poco a las pérdidas físicas y psicológicas que tendrán que experimentar. Estas tareas son: ajuste a la declinación de las fuerzas físicas y de la salud del cónyuge, ajuste a la pensión y reducción del salario, ajuste a los cambios en la salud del esposo, establecimiento de una afiliación explícita con el grupo coetáneo, adoptar y adaptarse flexiblemente a nuevos roles sociales y hacer adaptaciones físicas satisfactorias a la vivienda.

Según Newman y Newman (1979), la mayor transición de vida es la emergencia del individuo a la adultez. Todo lo que pasó antes fue preparación y todo lo que sigue después es actualización. Las tareas del adulto joven según señalan estos autores son: balance entre trabajo y placer, establecimiento de un vínculo de amistades con diversos grados de intimidad y la selección de actividades que reflejan los valores del individuo.

Para el adulto medio, en vez de mantener las destrezas adquiridas en la etapa anterior, Newman y Newman (1979) consideran que son tareas de este período: el manejo del hogar, valorar necesidades y habilidades, la evaluación de responsabilidades, organización del tiempo, planeamiento para el futuro y 
tomar decisiones. Este individuo medio, tiene la mayor potencialidad y creatividad para enfrentar los problemas de la vida, pero debe ser capaz de aceptar responsabilidad por sus actos. En la ádultez tardía, no importa su duración, el tema integrador es la búsqueda de sentido, por lo que se consideran sus tareas específicas, la aceptación de lo que se ha vivido, el desarrollo de un punto de vista sobre la muerte, así como reorientar las energías restantes hacia nuevos roles y actividades.

Para Erikson (1989) el teórico del ciclo vital más reconocido, las tareas de desarrollo adulto consisten en resolver los problemas psicosociales relevantes que en cada etapa se presentản en forma de crisis. Así, en la temprana adultez debe haber una evolución de la sexualidad hacia un compromiso interpersonal para resolver la tensión entre intimidad y soledad. En el adulto medio, la tarea es compleja y demanda trabajo continuado durante largo tiempo pues hay un aumento concreto de la responsabilidad por el bienestar de la siguiente generación y por las contribuciones que puede ofrecer a su familia y comunidad, de modo que resuelva la crisis de generatividad versus estancamiento. En la etapa final, propia del adulto viejo, la asignación consiste en alcanzar la integridad que sólo puede lograr después de pensar sobre el significado de la vida, aceptar la vida vivida y enfrentar la cercanía de la muerte sin horrorizarse. De esta manera, se soluciona la crisis de integridad versus desesperación. Estas crisis psicosociales pueden desembocar, según Erikson (1989) en su polo negativo: soledad, estancamiento y desesperación, pero eso indicaría que no se cumplieron adecuadamente las tareas asignadas.

Para Gould (1981), la tarea básica del periodo adulto es llegar a dominar los "demonios infantiles", o sea la cólera peligrosa que guardamos como un monstruo interno y que nos hace proceder a veces con injustificada frustración, egoísmo e irracionalidad. Esta tarea de la vida mental adulta es la "eliminación de las distorsiones de la conciencia infantil y sus demonios y de los mecanismos protectores que restringen nuestra vida" (Gould, 1978, p.27). Cuando se logra acabar con los monstruos infantiles, podemos abandonar las expectativas irrealizables las reglas inflexibles y los roles rígidos, así como superar ciertos supuestos fal- sos que cargamos desde niños. Los supuestos son: a.) siempre seré el niño de mis padres, b.) ellos siempre estarán para ayudarme cuando yo no pueda hacer algo, c.) la vida es simple y controlable y d.) no hay muerte real ni maldad en el mundo. Estos supuestos están relacionados de algún modo, con la edad y con la cultura en que vivimos, por lo que el individuo va superándolos gradualmente, y según su propia eficiencia para comprender y resolver material psicológico. Al hacerlo, se logra un cambio de conciencia y se abandonan mecanismos irracionales. Solo después se podrá alcanzar la libertad interna.

Un punto de vista distinto es el de Slavson (1969), quien destaca la importancia de las tareas humanas de dar y recibir. Recuerda cómo al nacer el niño es indefenso y para que sobreviva, los adultos que lo rodean deben cumplir la obligatoria asignación de: nutrirlo, informarlo y entrenarlo. El infante sólo pide y la madre le da con ternura. Es decir, el niño aquí recibe nada más. Conforme crece, se le empieza a disciplinar, se le ponen limitaciones, los adultos dejan de ser indulgentes con él y se inicia el entrenamiento para que aprenda a dar, pues esa será su futura tarea con la siguiente generación: darle nutrición, información y educación. Así se cierra el círculo en que primero se recibe para luego dar.

En síntesis, cada etapa de la vida requiere de ciertas asignaciones específicas, según la cultura. El periodo adulto no escapa a la inexorable ley de asumir responsablemente el ciclo vital individual, y por lo tanto, de cumplir con las tareas esperadas de la adultez.

\section{3. ¿Cuando termina una tarea?}

Así como en cada etapa de la vida la gente debe deshacerse de los roles que ya no son relevantes y enfocar sus energías en la continuación o la adquisición de nuevos roles -dejar de ser estudiantes, dejar de ser dependientes, de ser empleado, o soltero-así las tareas de crecimiento deben desaparecer como búsqueda personal, una vez que alcanzan su fin. Terminarlas a veces, produce confusión y ansiedad porque la persona puede quedar un poco en el vacío. Sin embargo, en la mayoría de los casos, cuando la tarea ha sido cumplida, el individuo genera otra necesidad propia de la 
nueva etapa de vida a que ingresa. Esto implica un trabajo nuevo, una tarea diferente, de modo que se perpetúa el patrón de cambio continuo y progresivo a través de la vida. Por eso la obligación central de vivir, es cambiar, sin mirar hacia el pasado, pero eso después de apreciar toda la sabiduría, la belleza, la inspiración que la historia propia ha proporcionado, y después de recoger todo lo que "se desea llevar" (Dyer, 1984). En esta constante transformación, cumplimos tareas de diferenciación primero y luego de integración, tareas de rebeldía y de aceptación, de recibir y de dar, de aprender y de crear, de planear y de construir, de amar y trabajar.

- La mayor parte de las veces es difícil evaluar las tareas que han sido complejas, mientras que hay algunas más concretas que son fáciles de realizar y también de valorar su cumplimiento. Para el adulto es importante saber que logró cumplir una tarea como para un corredor es importante marcar un mayor puntaje en su camino hacia la victoria. Algunos de estos logros son precipitados biológicamente, mientras que otros solo con cambios anticipados del rol social, pero siempre combinan aspectos biológicos, sociales y psicológicos de funcionamiento del ser humano.

La personalidad saludable no puede existir si el individuo se defiende reprimiendo su vitalidad,la sexualidad y otras emociones, pues ello produce tensión muscular, y destruye la capacidad para el placer (Jourard y Landsman, 1987). Tener sentido claro de la vida equivale a entregarse sinceramente a vivir y a hacer aquello que creemos que debemos y queremos hacer. De esta manera, todos sabremos cuándo terminamos una tarea, y si esa tarea fue eficaz y meritoria.

\section{4. ¿Cómo ayudar al adulto a aclarar y cumplir sus tareas?}

Para alcanzar los sueños y el potencial que acarreamos, es necesario superar el arcaico miedo a cambiar, y aceptar que la transformación del ser también puede traer tristeza profunda, pues dejamos atrás partes de nosotros mismos que ya no son útiles, que deben modificarse. De ahí que debamos cambiar. Esta es por lo mismo, la principal tarea adulta. Se espera que el cambio sea positivo y que pro- duzca desarrollo y crecimiento. El futuro es desconocido pero mucho de ese futuro ya está escrito porque nosotros seremos los que lo haremos. Si buscamos mejorar y crecer, ese futuro será mejor. Por supuesto, ese proceso no podrá entenderse fuera de la sociedad. En la determinación de la estructura que la persona da a su vida, la relación del pasado con el presente y con el medio económico-social es un factor poderoso que incidirá en las tareas que se pretenden cumplir.

Como decíamos, la estructura psicológica de la persona tiene sus inicios en las tempranas relaciones familiares que se modifican y solidifican luego con las experiencias de grupo del niño. "La forma como cuidemos de nosotros, las relaciones sociales que establezcamos, y la clase de actividades en que nos involucremos, nos llevarán directamente a la puerta de quiénes seremos en nuestros años tardíos", dijo Dychtwald (1990, p. XVII).

La actitud de los padres primero, y de los adultos en general después, influenciará la organización personal de cada individuo, y de alguna manera, va a predisponer a esa persona para el logro o fracaso en el cumplimiento de sus tareas. Por eso el objetivo al educar la siguiente generación, no es volverla sumisa y ansiosa, sino fortalecer su identidad para que alcance una mayor capacidad para dirigir sus propias vidas, para que ayuden a otros creando deliberados y positivos impactos en su futuro.

La orientación es parte consustancial de la educación. Los muchos y nuevos retos contemporáneos obligan a flexibilizar los diversos roles que han venido jugando los educadores y entre ellos, el orientador. Auguramos que su participación será predominante en esta era de incremento en la expectativa de vida de las personas, para ayudarlaś en la búsqueda de opciones de vida más flexibles, donde el plan de vida tradicional y lineal tiende a modificarse: hay una nueva dinámica emancipante en relación con los roles sexuales y con los estilos de convivencia y relación. El papel de las profesiones de ayuda seguirá siendo valioso en esta época de cambios culturales y sociopolíticos drásticos, a la vez que continuará la búsqueda directa por mayor bienestar y satisfacción individual. En esta realidad, la orientación puede ser vista como un instrumento de actualización, donde el orientador se usa a sí mismo 
como modelo para ayudar al cliente a trascender la vivencia cotidiana, a autorrealizarse. De esta manera, serán objetivos del orientador, lograr que el cliente gane comprensión de sí mismo, de los demás y del mundo, que alcance conductas positivas visibles y que sienta mayor satisfacción en su vida. Es decir, el papel del orienador será ofrecer ayuda sistemática y científica, no solo afectiva.

En el trabajo con adultos, sabemos también que es necesario ser conscientes del rol sexual y de la etapa que la persona está viviendo, así como de las tareas que la cultura específica demanda a cada uno de los períodos. Ayudar a un adulto significa permitirle reflexionar sobre sus problemas y la naturaleza compartida de muchos de ellos (Johnson, 1987), facilitarle la anticipación del futuro y al hacerlo, permitirle formular planes creativos para que desarrolle respuestas efectivas y controladas ante las diferentes situaciones de su vida (Collinson, 1981). Es decir, ayudarle a aclarar las tareas de desarollo que está intentando cumplir, y los diferentes medios que puede encontrar para realizarlas.

La manera de trabajar con los adultos en esta aclaración de intenciones y formulación de planes, varía: desde un esfuerzo de autoayuda individual,una relación interpersonal, hasta el trabajo en grupo. Creemos que la combinación de estos métodos es muy efectiva.

González (1980) encontró que existen cinco niveles diferentes para intervenir en el trabajo con adultos:

1. en la entrega de instrumentos básicos, como alfabetización,

2. en la formación profesional, como la capacitación,

3. en la instrucción social y cultural, como deportes y clubes artísticos,

4. en la educación cívica y política, como la que ofrecen los sindicatos y partidos políticos,

5. en lo relacionado con la maduración personal y familiar, donde aparecen los grupos de padres, de autoayuda y de orientación general.
Estos grupos de orientación, según Mc.Lennan y Fensenfeld (1969) pueden llevarse a cabo en cuatro áreas diferentes: 1) los que dan información, 2) sobre problemas cotidianos específicos para analizar sus ramificaciones y las alternativas posibles de solución, 3) los que buscan ajustes a crisis de desarrollo situacionales, que demandan cambios en las actitudes y el autoconcepto de las personas, y 4) los que se efectúan a nivel más informal, que ofrecen orientación indirecta pues se forman los grupos con propósitos de realizar una actividad específica, generalmente recreativa como clubes y talleres.

En estos niveles, se puede trabajar con técnicas directas para mejorar la vida, como la participación activa del individuo en lo que Brammer (1981) llama "intervención programada", que permite revisar los recursos con que cuenta el adulto en ese momento. Esta intervención no es para lidiar con problemas innatos sino con los que son propios del desarrollo, que requieren menos acomodo, o sea, cambios que no alteran totalmente el estilo de vida que la persona tenía. De ese modo no se produce un desequilibrio total. Otras estrategias auxiliares son muy valiosas, como la relajación, el autocontrol y el ejercicio físico, así como la anticipación de eventos con proyecciones al futuro.

Cuando se quiere descubrir la finalidad de la vida, encontrar el sentido y evitar así el tedio y el vacío existencial (Frankl, 1980), es útil recurrir a lo que Buscaglia (1986) ltamó "la búsqueda de plenitud y armonía", que permite encontrar planes para cambiarse a sí mismo y al mundo. Esto nos dará tareas y obligaciones. Encontraremos dentro de nosotros mismos, aquello que nos falta y así surgirá la esperanza que da dirección, fe, y tolérancia ante la incertidumbre. A esta búsqueda puede ayudar la relación de orientación individual, tanto como el trabajo grupal.

Jourard (1987) señala que la relación individual es insustituible, pero Knott (1987) considera importante sobre todo el trabajo grupal: de apoyo, terapia y autoayuda. Los grupos de ayuda son efectivos porque quien participa encuentra otras personas que buscan también superar dificultades en sus vidas, compartir preocupaciones y experiencias y porque permiten aprender por modelado, de 
los que son líderes y luchadores. Además, estos grupos se convierten en soporte social y de amistad. Los grupos de ayuda mutua permiten a sus miembros enfocar sus esfuerzos en mejorar la comunicación interpersonal, la autoafirmación, el entrenamiento de distintas destrezas que necesitan, proveyéndoles apoyo social para sus necesidades cognocistivas y afectivas, como confianza, validación, comparaciones intergeneracionales y alternativas de solución a sus dificultades y búsqueda (Teddler y Scherman, 1987).

Sabemos que es difícil que un adulto busque ayuda, por la presión social que afirma que los adultos deben ser poderosos. Ellos - tampoco quieren aceptar que a menudo se sienten confundidos y no saben hacia dónde van. Boice (1982) anota, sin embargo, que si se ofrece un servicio técnico que utilice primero las necesidades laborales específicas de esos adultos-apáticos y reticentes a la ayuda profesional-, muchos de ellos terminarán acercăndose al orientador.

Ciertamente la forma en que miramos el mundo da significado a las cosas, por lo que es necesario el replanteamiento constante para mirar cada vez con ojos nuevos lo que hacemos (Borysenko, 1990). Si los orientadores nos inmovilizamos obstinadamente, gastaremos energía en resistirnos al cambio y perderemos creactividad. Seremos como pájaros presos en una jaula que tiene la puerta abierta: es necesario arriesgarse a abandonar la seguridad del encierro para encontrar la libertad, la alegría y productividad. Solo así creceremos profesionalmente y cumpliremos con una tarea histórica de la Orientación.

Para explorar nuevos ámbitos laborales como el trabajo con adultos, necesitamos actitudés de compromiso y participación, de auto control o sea convicción de que podemos ejercer influencia sobre los acontecimientos, y de desafio, que es dejar de pensar que los cambios son amenazantes. Todo ello se podría encontrar en la fortaleza especial que es la búsqueda de transformación. Transformación de nosotros mismos, y renovación de lo que nos rodea. Así se cumple con una vida adulta llena de significado. ¡Esa es la mejor tarea que puede cumplir un orientador!

\section{Bibliografia}

Allport, Gordon. La persona en psicología. México: Trillas, 1988.

Boice, R. "Orientación de colegas" The personnel \& Guidance J.O. 1982.

Borysenko, Joan. Cómo alcanzar el bienestar físico y emocional mediante el poder de la mente. Bogotá: Norma, 1990.

Buscaglia, L. Bus 9 to paradise. New York: Fawcett Columbine, 1986.

Collinson, Brooke. "Orientación de adultos masculinos". The personall and guidance journal. 1981.

Dychtwald, K. \& Flower, Jo. Age Wave. Toronto: Bentam Books, 1990.

Dyer, W. El cielo es el límite. Barcelona: Grijalbo, 1984.

Erikson, E. Vital involvement in old age. Barcelona: Herder, 1980.

Frenkel, E. "Adjustments \& reorientation in the course of the life span" en Neugarten Middle age $E$ aging, 1968.

Gould, R.. Transformations" en Smelser y Erikson. Themes of work and love in adultbood, 1981.

Havighurst, R.J. Developmental tasks and education. New York: David Mckay, 1972.

Johnson, M. "Mental illness \& psychiatric treatment among women", en Roth Wallsh The psycbology of women. 1987.

Jourard \& Landsman. La personalidad saludable. México: Trillas, 1987.

Kermis, M. Mental bealth in late life. Boston: Jones and Barlette Pub. 1986.

Knott, J.G. "Grief work with men" en Scher et al. Counseling \& Psychotherapy with men, 1987. 
Lehr, Ursula. Psicología de la senectud. Barcelona: Herder, 1980.

Levinson, $\mathrm{D}$. The season's of a man's life. New York: Ballantine, 1978.

Mc.Lennan \& Felsenfeld Group Counseling $\varepsilon$ psychoterapy with adolescents. New York: Columbia University Press, 1969.

Newman y Newman. Development througb life. Illinois: Dorsey Press, 1979.

Nichols, Michael. Turning forty in the eightys. New York: Fireside Book, 1986.
Peck, R.C. "Psychological development in the 2nd. half of life", en Neugarten Middle age and aging, 1986.

Sheehy, C. Las crisis de la edad adulta. New York: Dutton \& Co, 1976.

Slavson, S.R. $A$ texbook in analytic group psychotherapy. New York: International University Press, 1969.

Tedder, S. y Scherman, A. "Counseling single fáthers" en Counseling \& Psicbotherapy with men, 1987. 